\title{
Estimation and Bias Correction of Aerosol Abundance using Data-driven Machine Learning and Remote Sensing
}

\author{
N. K. Malakar, D. J. Lary, A. Moore, \\ D. Gencaga, B. Roscoe \\ University of Texas at Dallas \\ Richardson, TX \\ nabin.malakar@utdallas.edu
}

\author{
Arif Albayrak, Maksym Petrenko, and Jennifer Wei \\ Goddard Earth Sciences DISC \\ NASA Goddard Space Flight Center \\ http://disc.gsfc.nasa.gov/
}

\begin{abstract}
Air quality information is increasingly becoming a public health concern, since some of the aerosol particles pose harmful effects to peoples health. One widely available metric of aerosol abundance is the aerosol optical depth (AOD). The AOD is the integrated light extinction coefficient over a vertical atmospheric column of unit cross section, which represents the extent to which the aerosols in that vertical profile prevent the transmission of light by absorption or scattering. The comparison between the AOD measured from the ground-based Aerosol Robotic Network (AERONET) system and the satellite MODIS instruments at $550 \mathrm{~nm}$ shows that there is a bias between the two data products. We performed a comprehensive analysis exploring possible factors which may be contributing to the inter-instrumental bias between MODIS and AERONET. The analysis used several measured variables, including the MODIS AOD, as input in order to train a neural network in regression mode to predict the AERONET AOD values. This not only allowed us to obtain an estimate, but also allowed us to infer the optimal sets of variables that played an important role in the prediction. In addition, we applied machine learning to infer the global abundance of ground level PM2.5 from the AOD data and other ancillary satellite and meteorology products. This research is part of our goal to provide air quality information, which can also be useful for global epidemiology studies.
\end{abstract}

\section{INTRODUCTION}

Atmospheric aerosols are tiny particles (solid and liquid) suspended in the atmosphere. Some aerosols pose harmful effects to peoples health when inhaled. Moreover, atmospheric aerosols play an important role in understanding the global climate.

The aerosol optical depth (AOD), or optical thickness, is defined as the integrated extinction coefficient over a vertical column of unit cross section. The Extinction coefficient is the fractional depletion of radiance per unit path length and represent how much aerosols prevent the transmission of light by absorption and scattering.

In the past, much effort has been placed in observing aerosol characteristics, such as AOD, from space and ground-based instruments. The Moderate Resolution Imaging Spectroradiometer (MODIS), onboard the Terra and Aqua satellites, retrieve AOD using dark target methods in bands at 550, 670,870, 1240, 1630, and $2130 \mathrm{~nm}$ over the ocean, and at 470, 550, and $670 \mathrm{~nm}$ over land [1], [2]. A global system of ground-based sun and sky scanning sun photometers, called the Aerosol Robotic Network (AERONET), also measure the AOD at various wavelengths (at 340, 380, 440, 550, 675, 870, and $1020 \mathrm{~nm}$ ) [3]. AERONET measurements are taken every $15 \mathrm{~min}$ during daylight, and its level 2 quality control measurements assure AOD observations are accurate to within 0.01 for wavelengths of $440 \mathrm{~nm}$ and higher. AOD measurements from MODIS are available globally, whereas AERONET measurements are available only for land locations, some of which are coastal sites.

Ideally, the measurements of AOD from these two instruments should match. However, biases do exist between AERONET and MODIS measurements. In this study when we refer to the difference between the ground truth AERONET AOD observations at $550 \mathrm{~nm}$ and the remotely sensed MODIS AOD at $550 \mathrm{~nm}$ as the bias, i.e.

Bias = AEORNET AOD at $550 \mathrm{~nm}-$ MODIS AOD at 550 $\mathrm{nm}$. The bias is higher for higher AOD values. Figure 1 shows that a significant number of points do not fall close to the 1:1 line. Figure 2 shows that the magnitude of the bias is greater for larger values of AOD at $550 \mathrm{~nm}$. Our goal is to try and understand the factors that can delineate these extrema, and /or explain them statistically.

\section{Previous Studies}

Previous MODIS aerosol validation studies compared the Aqua and Terra MODIS-retrieved AODs with the groundbased AERONET observations [4], [5], [2], [6].

From the studies of Normalized Difference Vegetation Index (NDVI), Brown et al. (2008) suggested that the surface type played a key role in explaining a significant fraction of the observed bias [7].

Lary et al. (2009) used machine-learning approaches to explore factors contributing to a persistent bias between AOD retrieved from MODIS and AERONET data [8]. Their work also suggested a link between the MODIS AOD bias and the surface type. The possible factors influencing the 


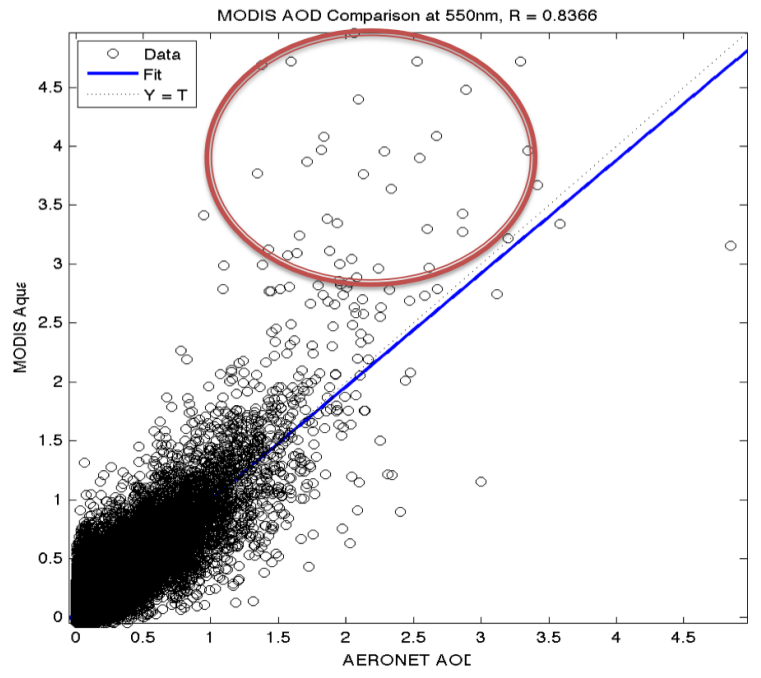

Figure 1. A scatter diagram showing the comparison between the AOD from AERONET and MODIS instruments at $550 \mathrm{~nm}$. The regime of high bias has been indicated by a circle.

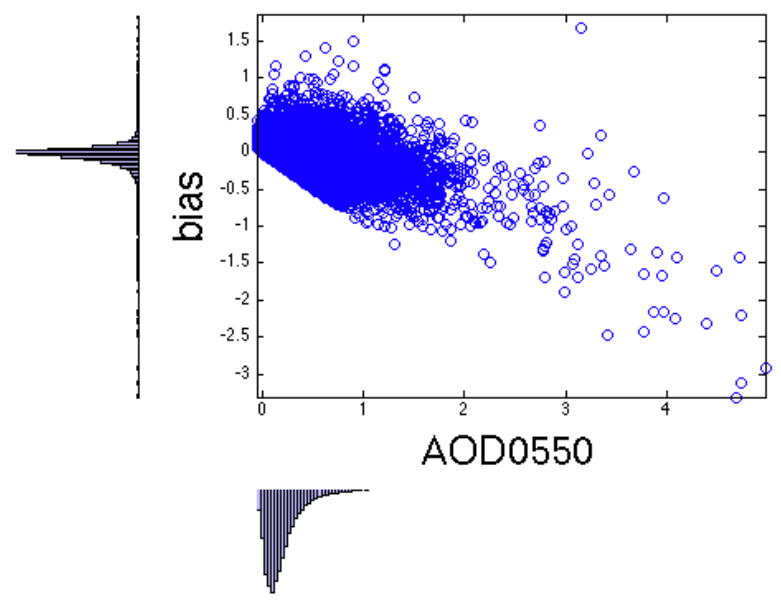

Figure 2. Scatter-histogram showing the distribution of bias and MODIS AOD measurements at $550 \mathrm{~nm}$.

bias might be associated with the measurement conditions such as the solar and sensor zenith angles, the solar and sensor azimuth, scattering angles, and surface reflectivity at the various measured wavelengths, etc. In their study they explained the AOD bias between MODIS and AERONET by using the surface type, the solar zenith angle, the solar azimuth angle, the sensor zenith angle, the sensor azimuth angle, the scattering angle, and the reflectance at $550 \mathrm{~nm}$ as input variables to the neural network.

In this paper, we performed a comprehensive analysis for every possible combination of the variables as input to train the neural network in regression mode to predict the AOD values. We then compared how well the predictions matched with the observed AOD values. As a result we obtained

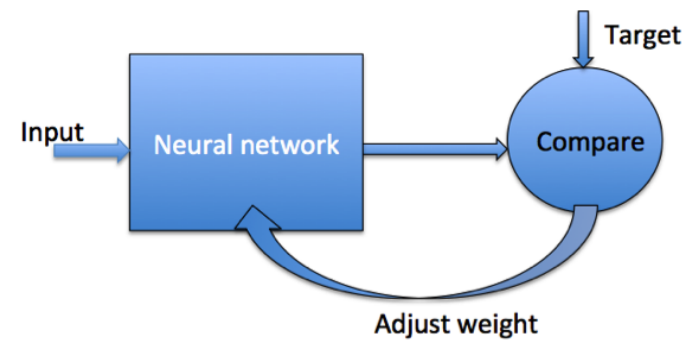

Figure 3. Supervised neural network technique.

the best set of variables explaining the bias in the MODIS (AOD) measurements.

\section{Neural Network Regression Technique}

Neural networks (NN) are biologically inspired algorithms used for classification or function approximation [9], [10], [11]. NNs are widely used in pattern recognition, machine learning and artificial intelligence. In addition, NNs have found many applications in other fields such as geoscience, remote sensing, oceanography, etc. Neural networks are also referred to as a multi-layer perceptron method because they may consist of multiple layers (e.g., input, hidden and output layers). Each neuron is connected to all other neurons in the adjacent layers. Each of neuron is assigned weights for each interconnection with other neuron. The output of the $k^{t h}$ neuron can be written as the weighted sum of inputs:

$$
y_{k}=\varphi\left(\sum_{j=1}^{n} w_{k j} x_{j}\right),
$$

where $\varphi$ is the transfer function, $w_{k j}$ represents the weight from unit $j$ to unit $k$ and $x_{j}$ represents the m input variables to the neuron. During training the NN weights are adjusted appropriately to learn the data. The learning, and adjustments of the weights are inspired by the synaptic learning behavior of neurons.

For an observation data set with $\mathrm{n}$ input variables, say $\left\{x_{1}, x_{2}, x_{3}, \ldots, x_{n}\right\}$, the observed output variable, AOD, is some function of these input variables. Our approach approximates the function by non-parametric, non-linear NNs. We selected supervised NN method since a NN learns from its input parameters, and is free from assumptions about its inputs. This allows us to explore various sets of inputs. The goal here is to train the NN against the AERONET AOD data as the target as shown in the figure 3 . The trained $\mathrm{NN}$ is then used to predict the AOD for the given set of variables.

As we describe next, we applied a neural network regression technique to learn the inter-instrumental bias and seek the best set of variables contributing to the bias. 


\section{SEARCh FOR OPTIMAL SET OF VARIABLES FOR Bias REDUCTION}

We observed AOD at $550 \mathrm{~nm}$ along with 14 other variables that are listed below. For brevity, we have denoted the variables by the corresponding numbers in the tables that follow.

1) Aerosol optical depth at $550 \mathrm{~nm}$ (AOD0550)

2) Aerosol optical depth at $470 \mathrm{~nm}$ (AOD0470)

3) Aerosol optical depth at $660 \mathrm{~nm}$ (AOD0660)

4) Mean reflectance at $470 \mathrm{~nm}(\mathrm{mref0470})$

5) Mean reflectance at $550 \mathrm{~nm}(\mathrm{mref0550)}$

6) Surface reflectance at $660 \mathrm{~nm}$ (surfre0660)

7) Surface reflectance at $660 \mathrm{~nm}$ (surfre0470)

8) Surface reflectance at $660 \mathrm{~nm}$ (surfre2100)

9) Cloud fraction from land aerosol cloud mask (cfrac)

10) Quality assurance (QAavg)

11) Solar zenith angle (SolarZenith)

12) Solar azimuth angle (SolarAzimuth)

13) Viewing zenith angle (SensorZenith)

14) Sensor azimuth angle (SensorAzimuth)

15) Scattering angle (ScatteringAngle)

The number of combinations for $\mathrm{n}$ variables, considering a set of $\mathrm{k}$ at a time, is given by the combination ${ }^{n} C_{k}$. Our AOD data set contains 15 measured variables and, we considered all the possibilities such as ${ }^{15} C_{15},{ }^{15} C_{14},{ }^{15} C_{13},{ }^{15} C_{2}$. Thus, there are 32,781 possible combinations to be explored. So, we have made this a search problem where the search is over the possible set of variables that can best fit the observed data. At end, the non-relevant variables will be absent from the best fitting set of variables.

For each combination set, one at a time, we trained the NN with AERONET AOD as the target variable, and then predicted what this AERONET AOD is from the trained network. The NN algorithm used a feed-forward back propagation algorithm with a hidden layer having 200 nodes as shown in figure 4 . The training was done by the Levenberg-Marquardt algorithm with mean-squared error as the performance factor provided by the Matlab NN toolbox. When training a neural network, we randomly split the training data set into three portions, in the ratio of $80: 10: 10$. The first $80 \%$ portion is used to train the NN weights using an iterative process. For each iteration, we evaluate the current root mean square (RMS) error of the neural network by using the second $10 \%$ portion of the data (this portion was not used in the training). We use the RMS error and how it changes with the training iterations (known as epochs) to determine the convergence of our training. When the training is complete, we use the final $10 \%$ of the data as the validation data set.

Since the neural network constructs a mapping between the set of input variables and the output variables. The most relevant set of variable is the one that can best reproduce the target data. We explored all combinations of variables, which

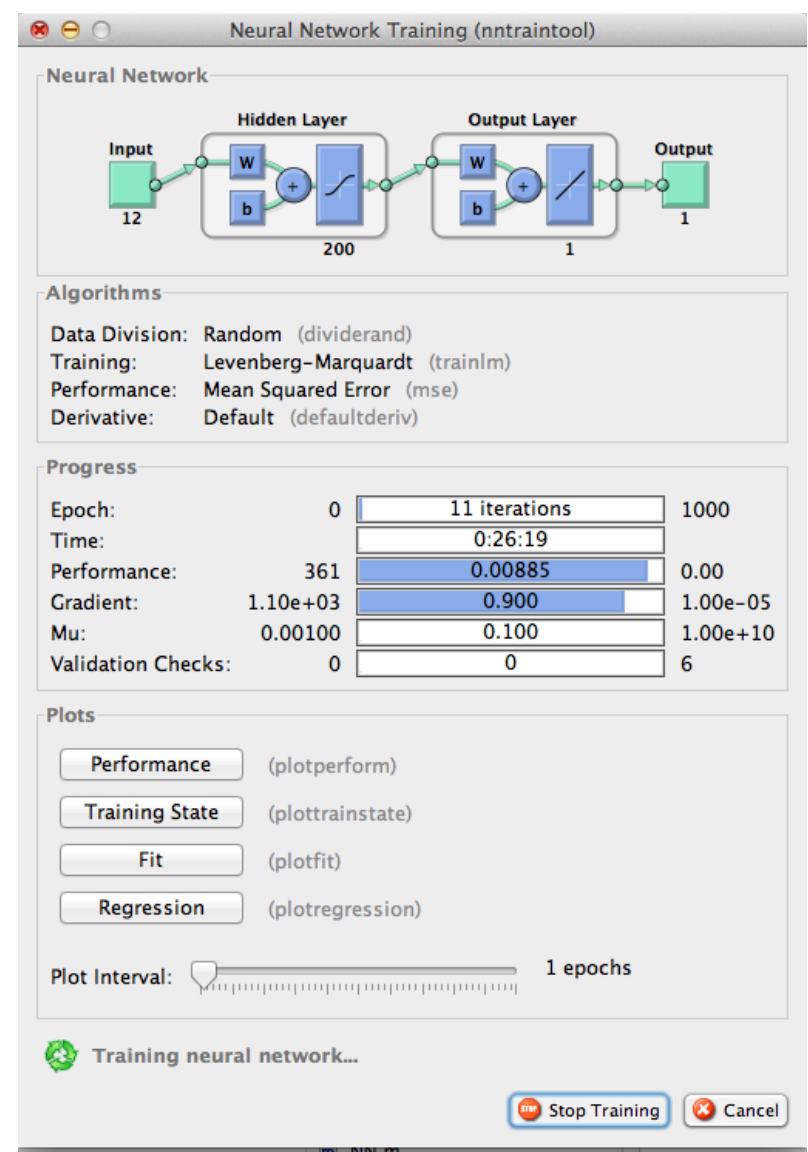

Figure 4. Matlab's neural network toolbox was used to train the neural nets. A screenshot of 12 variable training case is shown.

provided the fit of the observed AERONET AOD data. The end product is a regression between the available satellite variable, which are used to predict the observed AERONET AOD. This is a massive number crunching exercise. We automated the workflow for each combination by writing a job-parallel code.

\section{Similarity measure Between PREDicted AND OBSERVED AOD}

In order to quantify the agreement between the observed and predicted data, we used both the correlation coefficient appropriate for Gaussianly distributed variables and the mutual information appropriate for variables of arbitrary probability distribution. The predictions made by the most relevant set of variables show the highest correlation coefficient or highest mutual information with the observed data. In the appendix, we show that Mutual Information (MI) is the more general case of correlation coefficient. When we assume the normal distribution, the expression of MI returns the correlation measure. So, it makes sense to use MI as the general measure of correlation between the observed and predicted set as many of the variables are not normally 


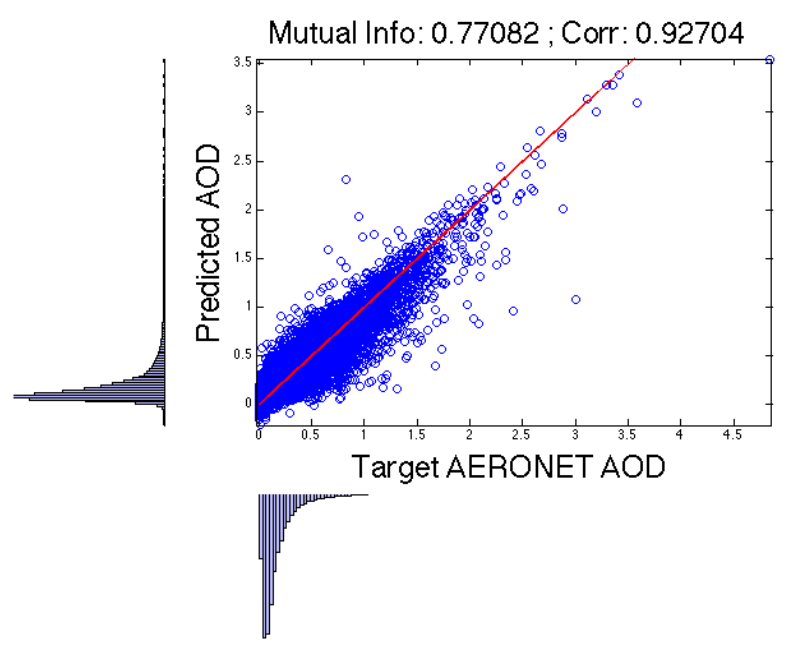

Figure 5. Scatter diagram showing the comparison between the AOD from AERONET and the NN correction. The NNs are able to learn and address the bias correction. Similar bias correction from the application of $\mathrm{NN}$ has been extended to explore all the possible combination of variables. Table 1 shows that the highest set of relevant variable consists of only 14 variables, as opposed to the complete set of 15 variables.

distributed.

In the literature, there are several methods to estimate MI from data [12], [13], [14]. We applied the variable bin width histogram approach [15], [16] to compute the normalized MI between the observed and predicted AOD. Higher values indicate better agreement between the observed and predicted set, and thus, are the best indicators of the input variables needed to assess a relevant set of variables. We compiled a table containing the MI for all sets in the decreasing order and are presented in Table I.

We note that we can construct the best regression fit of the MODIS parameters to predict the AERONET AOD when certain MODIS parameters are absent in the combination. Table II shows the absent variables from the combination. These absent variables include Aerosol optical depth at 550 $\mathrm{nm}$ (AOD0550), AOD at $660 \mathrm{~nm}$, Cloud fraction from land aerosol cloud mask (cfrac), Surface reflectance at $470 \mathrm{~nm}$ (surfre0470), Surface reflectance at $660 \mathrm{~nm}$ (surfre0660), Sensor azimuth angle (Sensor Azimuth), Solar Zenith angle (Solar Zenith) etc. The neural networks performed better or could reproduce the observed AOD data in the absence of certain variables indicates that presence of the aforementioned variables in the NN input attribute to the observed bias.

Therefore, the methodology of comprehensive search provides us insights into the factors explaining the bias between the MODIS AOD and AERONET AOD, and we also obtain the best performing NN which can then be used to estimate the bias corrected AOD observations [17]. Figure 5 shows the bias corrected AOD plot compared to the AERONET AOD. Clearly, the bias at the higher values of the AOD
Table I

TABLE SHOWING THE MI AND CORR-COEFF VALUES BETWEEN OBSERVED AND PREDICTED AOD. THE TABLE IS ARRANGED IN DESCENDING ORDER OF MI VALUES FOR VARIOUS COMBINATION OF INPUT VARIABLES.

\begin{tabular}{|lcc|}
\hline Combination & Mutual Information (MI) & Corr-Coeff(r) \\
\hline 23456789101112131415 & 0.771 & 0.927 \\
\hline 12456781011121315 & 0.769 & 0.926 \\
\hline 1234568101112131415 & 0.768 & 0.926 \\
\hline 1245689101112131415 & 0.766 & 0.926 \\
\hline 12345678910121315 & 0.765 & 0.926 \\
\hline 1245781012131415 & 0.764 & 0.925 \\
\hline 124567891011121314 & 0.762 & 0.921 \\
\hline 23456781011121314 & 0.761 & 0.924 \\
\hline 1345678101112131415 & 0.760 & 0.924 \\
\hline 124578101112131415 & 0.759 & 0.925 \\
\hline 124567101112131415 & 0.759 & 0.925 \\
\hline 134567891011121315 & 0.756 & 0.924 \\
\hline 1245681011121315 & 0.756 & 0.921 \\
\hline 1245781011121315 & 0.755 & 0.923 \\
\hline 23457891011121315 & 0.755 & 0.924 \\
\hline 12345678910111215 & 0.755 & 0.921 \\
\hline 12345789101112131415 & 0.754 & 0.875 \\
\hline 13456791011121315 & 0.754 & 0.924 \\
\hline 1345681012131415 & 0.753 & 0.922 \\
\hline 234569101112131415 & 0.753 & 0.923 \\
\hline
\end{tabular}

Table II

ABSENT VARIABLES IN TABLE 1

\begin{tabular}{|ll|}
\hline Row \# & Absent variables in the set as shown in the Table 1 \\
\hline $\mathbf{1}$ & AOD 0550 \\
\hline $\mathbf{2}$ & AOD0660, cfrac, SensorAzimuth \\
\hline $\mathbf{3}$ & surfre0470, cfrac \\
\hline $\mathbf{4}$ & AOD0660, surfre0470 \\
\hline $\mathbf{5}$ & SolarZenith, SensorAzimuth \\
\hline $\mathbf{6}$ & AOD0660, surfre0660, cfrac, SolarZenith \\
\hline $\mathbf{7}$ & AOD0660, ScatteringAngle \\
\hline $\mathbf{8}$ & AOD0550, cfrac, ScatteringAngle \\
\hline $\mathbf{9}$ & AOD0470, cfrac \\
\hline $\mathbf{1 0}$ & AOD0660, surfre2100, cfrac \\
\hline
\end{tabular}

have been corrected and the AOD values follow values close to $1: 1$ line. This best prediction was obtained by a set consisting of the following variables: AOD at $470 \mathrm{~nm}$, and $\mathrm{AOD}$ at $660 \mathrm{~nm}$, Mean reflectance at $470 \mathrm{~nm}$, and Mean reflectance at $550 \mathrm{~nm}$, Surface reflectance at $660 \mathrm{~nm}, 470$ $\mathrm{nm}, 2100 \mathrm{~nm}$, Cloud fraction, Quality assurance values, Solar zenith angle, solar azimuth angle, Zenith angle, Sensor azimuth angle and scattering angle.

\section{Estimating Global PM2.5 ABUndance}

We also applied machine learning to infer the global abundance of ground level distribution of particles with a diameter of 2.5 micrometers (PM 2.5) or less from the AOD data and other ancillary satellite and meteorology 


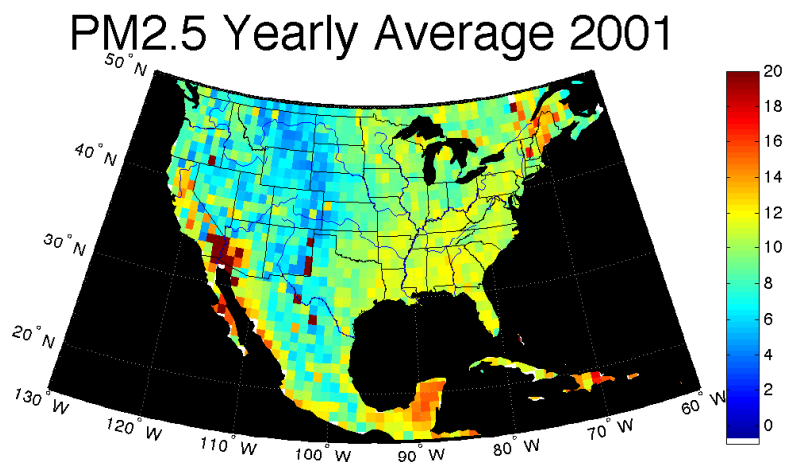

Figure 6. The yearly average PM 2.5 distribution for continental USA, generated from satellite data, weather analysis, and roadside GLP observations.

products. The abundance of PM2.5 at ground level is known to adversely impact public health. For example, it is known to have serious impacts on people with hearth diseases, asthma, or some cardiovascular diseases, etc [18].

Ground monitoring stations are only available at certain locations, so we do not have in-situ observations of ground level PM2.5 (GLP) for the whole planet. It is not always possible to obtain the GLP in the rapidly increasing population area, which are also the newer locations of air pollution. However, if we can use remotely sensed data we can provide a daily GLP data product for the entire globe. By constructing an automated workflow with large computing facilities, it will be possible to examine the GLP for any location in the world and also to analyze the trend of changes in global air pollution.

Figure 6 shows the GLP for the continental USA generated from satellite data, weather analysis, and roadside GLP observations. Currently there is a full coverage of ground level PM2.5 in the US, and nearly full global coverage. The estimation of GLP has important health application. Since the GLP products can be used to construct a global air pollution map, one can construct an application for personal digital assistant (PDA) advising the adverse outdoor situation. We have implemented such an approach and this is work in progress.

The global estimation with a continuous spatial and temporal coverage can be critically useful in making health care decisions. This will also help in making public policy decisions for improving the GLP and environmental conditions.

\section{CONClusions AND Future Works}

In this paper, we studied factors influencing the bias in the observed AOD values between the MODIS and AERONET instruments. We applied supervised neural network method in regression mode to train the NN with AERONET data set as the target, and recomputed the prediction of AOD from the neural nets. We performed an exhaustive search for the possible combinations of input variables.
The best prediction of AOD, which had maximum mutual information with AERONET, was provided by the set consisting of the following variables: AOD at $470 \mathrm{~nm}, 660 \mathrm{~nm}$, Mean reflectance at $470 \mathrm{~nm}, 550 \mathrm{~nm}$, Surface reflectance at 660nm, $470 \mathrm{~nm}, 2100 \mathrm{~nm}$, Cloud fraction, Quality assurance values, Solar zenith angle, solar azimuth angle, Zenith angle, Sensor azimuth angle and scattering angle. The best agreement between the observed and predicted AOD occurred when some of the variables were missing from the input combinations. For example, the best set is missing AOD $550 \mathrm{~nm}$ itself in the set i.e the neural network performes best in the absence of MODIS AOD values at $550 \mathrm{~nm}$. Similarly, for various other combinations, the absence of one or couple of variables such as the AOD at $660 \mathrm{~nm}$, the cloud fraction from the land aerosol cloud mask, the surface reflectance at $470 \mathrm{~nm}$ and at $660 \mathrm{~nm}$, the sensor azimuth angle, the solar zenith angle etc. seems to indicate that their presence can be attributed to the observed bias.

The method of estimating the AOD was also applied to estimate the ground level PM2.5, which are known to have adverse health effects. In this case we used the AOD measurements and ancillary information as input and trained the NN in regression mode to estimate the GLP. The global estimation with a continuous spatial and temporal coverage can critically help in making public policy decisions for improving the GLP environmental conditions and healthcare decisions.

\section{APPENDIX}

Correlation coefficient (Pearsons correlation) is a widely used measure of dependence between two variables, and represents the normalized measure of the strength of their linear relationships. The correlation coefficient $\rho_{X, Y}$ between two random variables $X$ and $Y$ with expected values $\mu_{X}$ and $\mu_{Y}$ and standard deviations $\sigma_{X}$ and $\sigma_{Y}$ is defined as:

$$
\rho_{X, Y}=\frac{\operatorname{cov}(X, Y)}{\sigma_{X} \sigma_{Y}}=\frac{E\left[\left(X-\mu_{X}\right)\left(Y-\mu_{Y}\right)\right]}{\sigma_{X} \sigma_{Y}}
$$

where, $\mathrm{E}$ is the expected value operator, cov means covariance, and, $\rho$ a widely used alternative notation for Pearson's correlation.

The correlation coefficient is defined only if both of the standard deviations are finite and both of them are nonzero. The correlation coefficients range from -1 to 1 . The correlation coefficient values close to 1 , (or -1) suggest that there is a positive (or negative) linear relationship between the data columns, whereas the values close to or equal to 0 suggest there is no linear relationship between the data columns. It can only be applied to the cases of linear relationship between two variables.

Mutual information quantifies the mutual dependence between two variables, by taking into account the whole probability distribution function (PDF) characteristics of the variables. Mutual information (MI) is defined as follows in 
discrete form:

$$
I(X, Y)=\sum_{x \in X} \sum_{y \in Y} p(x, y) \log \frac{p(x, y)}{p(x) p(y)},
$$

which is a special case of a measure called KullbackLeibler divergence [19], [20]. If $X$ and $Y$ are statistically independent, then

$$
p(x, y)=p(x) p(y) .
$$

In this case, the mutual information becomes 0 , showing independency. A proper mapping of the form

$$
\delta(X, Y)=\sqrt{1-e^{-2 I(X, Y)}}
$$

normalizes the measure of general correlation as depicted by the MI [21], [22], [23]. In the case when $\mathrm{X}$ and $\mathrm{Y}$ are normally distributed,

$$
(X, Y) \sim \mathcal{N}(\mu, K)
$$

where, $K=\left(\sigma^{2}, \rho \sigma^{2} ; \rho \sigma^{2}, \sigma^{2}\right)$. The mutual information reduces to

$$
I(X, Y)=-\frac{1}{2} \log \left(1-\rho^{2}\right) .
$$

So, that,

$$
\delta(X, Y)=\sqrt{1-e^{-2 I(X, Y)}}=|\rho(X, Y)| .
$$

This relation shows the generality of the normalized correlation measure.

\section{REFERENCES}

[1] Y. Kaufman, D. Tanré, L. Remer, E. Vermote, A. Chu, and B. Holben, "Operational remote sensing of tropospheric aerosol over land from EOS moderate resolution imaging spectroradiometer," J. Geophys. Res, vol. 102, no. 17, pp. 17,051-17,067, 1997.

[2] L. Remer, R. Kleidman, R. Levy, Y. Kaufman, D. Tanré, S. Mattoo, J. Martins, C. Ichoku, I. Koren, H. Yu et al., "Global aerosol climatology from the MODIS satellite sensors," J. Geophys. Res, vol. 113, no. D14, p. S07, 2008.

[3] B. Holben, T. Eck, I. Slutsker, D. Tanré, J. Buis, A. Setzer, E. Vermote, J. Reagan, Y. Kaufman, T. Nakajima et al., "Aeroneta federated instrument network and data archive for aerosol characterization," Remote Sensing of Environment, vol. 66, no. 1, pp. 1-16, 1998.

[4] D. Chu, Y. Kaufman, C. Ichoku, L. Remer, D. Tanré, and B. Holben, "Validation of modis aerosol optical depth retrieval over land," Geophys. Res. Lett, vol. 29, no. 12, p. 8007, 2002.

[5] L. Remer, Y. Kaufman, D. Tanré, S. Mattoo, D. Chu, J. Martins, R. Li, C. Ichoku, R. Levy, R. Kleidman et al., "The MODIS aerosol algorithm, products, and validation," Journal of the Atmospheric Sciences, vol. 62, no. 4, pp. 947-973, 2005.
[6] R. Levy, L. Remer, S. Mattoo, E. Vermote, and Y. Kaufman, "Second-generation operational algorithm: Retrieval of aerosol properties over land from inversion of moderate resolution imaging spectroradiometer spectral reflectance," $J$. Geophys. Res, vol. 112, no. D13, p. D13, 2007.

[7] M. Brown, D. Lary, A. Vrieling, D. Stathakis, and H. Mussa, "Neural networks as a tool for constructing continuous NDVI time series from AVHRR and MODIS," International Journal of Remote Sensing, vol. 29, no. 24, pp. 7141-7158, 2008.

[8] D. Lary, L. Remer, D. MacNeill, B. Roscoe, and S. Paradise, "Machine learning and bias correction of MODIS aerosol optical depth," Geoscience and Remote Sensing Letters, IEEE, vol. 6, no. 4, pp. 694-698, 2009.

[9] R. Lippmann, "An introduction to computing with neural nets," ASSP Magazine, IEEE, vol. 4, no. 2, pp. 4-22, 1987.

[10] S. Haykin, Neural Networks: A Comprehensive Foundation. Macmillan Coll Div, Jan. 1994.

[11] C. M. Bishop, Neural Networks for Pattern Recognition, 1st ed. Oxford University Press, USA, Jan. 1996.

[12] Y. Moon, B. Rajagopalan, and U. Lall, "Estimation of mutual information using kernel density estimators," Physical Review $E$, vol. 52, no. 3, pp. 2318-2321, 1995.

[13] D. N. Reshef, Y. A. Reshef, H. K. Finucane, S. R. Grossman, G. McVean, P. J. Turnbaugh, E. S. Lander, M. Mitzenmacher, and P. C. Sabeti, "Detecting novel associations in large data sets," Science, vol. 334, no. 6062, pp. 1518-1524, 2011.

[14] D. Gencaga, N. K. Malakar, and D. J. Lary, "Survey on the estimation of mutual information methods as a measure of dependency versus correlation analysis," to be published.

[15] G. Darbellay and I. Vajda, "Estimation of the information by an adaptive partitioning of the observation space," Information Theory, IEEE Transactions on, vol. 45, no. 4, pp. 13151321, 1999.

[16] P. Tichavsky, "Matlab code for estimating mutual information." [Online]. Available: http://si.utia.cas.cz/downloadPT.htm

[17] N. K. Malakar, D. Gencaga, and D. J. Lary, "Towards identification of relevant variables in the observed aerosol optical depth bias between modis and aeronet observations," to be published.

[18] http://www.epa.gov/pm/health.html.

[19] S. Kullback, Information theory and statistics. Dover Pubns, 1997.

[20] T. Cover and J. Thomas, Elements of Information. John Wiley \& Sons, New Jersey, 2006.

[21] H. Joe, "Relative entropy measures of multivariate dependence," Journal of the American Statistical Association, pp. 157-164, 1989. 
[22] C. Granger and J. Lin, "Using the mutual information coefficient to identify lags in nonlinear models," Journal of Time Series Analysis, vol. 15, no. 4, pp. 371-384, 1994.

[23] A. Dionisio, R. Menezes, and D. A. Mendes, "Mutual information: a measure of dependency for nonlinear time series," Physica A: Statistical Mechanics and its Applications, vol. 344, no. 12 , pp. $326-329,2004$. 